\title{
Cysteine as a potential donor urinary biomarker for donor acute kidney injury and recipient early graft function
}

\author{
Chris Tae Young Chung, Hyunmin Ko, Hyo Kee Kim, Kwangwoo Choi, Ahram Han, Sangil Min, Jongwon Ha
}

Division of Transplantation, Department of Surgery, Seoul National University Hospital, Seoul, Korea

Background: In deceased donor kidney transplantation, there is an increase in the use of marginal kidneys from donors with acute kidney injury with higher chance of delayed graft function (DGF). N-acetyl cysteine, a prodrug to cysteine and also a precursor to intracellular antioxidant glutathione, is known to have protective effects in different models of ischemic reperfusion injury. In this study, we aim to investigate cysteine as a potential donor urinary biomarker to predict acute kidney injury in donors and early graft function in recipients.

Methods: Kidney transplant recipients from deceased donors with urine samples at a single institution from 2015 to 2019 were included. Cysteine analysis was performed using a cysteine targeting fluorescence probe from previous studies. Donor risk factors associated with donor acute kidney injury (AKI) and short term outcomes such as DGF, acute rejection and graft function over time were analyzed using multivariate logistic regression.

Results: Ninety-one patients who underwent deceased donor kidney transplantation had donor urine samples. The mean fluorescent intensity of cysteine in urine samples from these cadaver donors was higher compared to that of normal living donors (3,145 vs. $687.3 \mathrm{AU} ; \mathrm{P}<0.001)$. In the multivariate analysis, donor factors significantly associated with $\mathrm{AKI}$ of donors were final serum creatinine before organ procurement, cold ischemic time and cysteine level $<1,500$. Within the donor AKI group, cysteine level >1,500 was associated with acute rejection in the protocol biopsy at postoperative day 10 .

Conclusions: This study provides the hypothesis that cysteine levels in cadaver donors increase in response to ischemic damage and decreases in order to reduce ischemic damage in donors with AKI. Donor urinary cysteine could be a potential predictive factor for donor AKI, early acute rejection and possibly DGF with the support of future in vivo and clinical studies.

Corresponding author: Chris Tae Young Chung

E-mail: tylight8@gmail.com

(C) The Korean Society for Transplantation

This is an Open Access article distributed under the terms of the Creative Commons Attribution Non-Commercial License (http://creativecommons.org/licenses/by-nc/4.0/) which permits unrestricted non-commercial use, distribution, and reproduction in any medium, provided the original work is properly cited. 